\title{
Nobel thoughts
}

\author{
The community of scientists should celebrate the Nobel Prize, even if awards bestowed \\ on one discipline are associated with another discipline. A new prize might help.
}

At a time when scientific institutions are struggling to maintain financing, the Nobel Prizes in Chemistry, Physics, and Physiology or Medicine stand out as invaluable symbols of the long-term value of basic and applied research. Every fall, they focus media attention on seminal advances with a profound impact on science and society and highlight the names, the faces andoccasionally - the human stories behind the research.

As a journal devoted to methods, we cannot resist pointing out that this year's prizes again confirm the vital importance of method and tool development. The chemistry prize was awarded for methods using palladium for synthesis of complex carbon-based molecules. The prize in medicine recognized the impact of the in vitro fertilization method on society. And graphene, the subject of the physics prize, is almost certain to be a powerful tool for many applications, including in biology.

The prominent role of methods in the prizes goes back many years. Well over a third of physics and chemistry prizes have had a methodological aspect to them, and many of these methods-X-ray spectroscopy, phase-contrast microscopy, mass spectroscopy, PCR and GFP, to name a few-have been seminal for biological research. In the past 10 years, half the medicine prizes featured important methodologies.

The awarding of prizes in chemistry to advances associated with biology, however, has resulted in a backlash among chemists. Complaints appeared to reach new levels last year when the prize was awarded to three scientists for work on the ribosome. An editorial in Nature Chemistry even commented on the community's dissatisfaction with the award. But non-biology prizes awarded for biological relevance will become more common; the proportion of biological research continues to expand relative to other fields, and innovations in physics and chemistry are increasingly applied to biology.

Obviously no prize system is perfect, particularly when the determinations must be subjective, but the Nobel selection process-based on Alfred Nobel's original stipulations-is intended to obtain broad expert support. This involves multiple nomination routes, including from experts around the world, followed by an evaluation of the nominees by separate Nobel committees for each prize. Aided by outside experts, the committees winnow the nominees down to about 15 candidates per prize. The final selection of the prizes for chemistry and physics is determined by a vote of the entire Royal Swedish Academy of Sciences, including biologists. The prize for physiology or medicine is decided by the Nobel Assembly of approximately 50 professors in medical subjects at the Karolinska Institute.

Thus, the Nobel system is set up to award accomplishments that advance the wider scientific community, and that community is increasingly tipped toward biology. US government statistics show that three times more $\mathrm{PhDs}$ have recently been awarded in biology than in chemistry, and the spread is greater for physics. Research funding patterns suggest this underestimates the divide in the number of active researchers in each area. Although the amount of Nobel Prize-worthy work going on in each area is impossible to estimate, the numbers will continue to favor biology. Still, if the chemistry community decided there was continual, unjustified bias for biology, that dissatisfaction could affect the process and erode support for the prizes.

So why not a Nobel Prize in Biology? When Alfred Nobel drafted his will, biomedical research was in its infancy, and he could not have envisioned the scope of the life sciences today. Not only are biologists working to elucidate the mechanisms of the cell at the level of single molecules, they are also attempting to discern the function and environmental role of entire ecosystems.

In 1968 the Nobel Foundation did allow a donation for the establishment of an award in economics but decided against future new prizes. The foundation is clearly obligated to administer the Nobel Prizes as dictated by Alfred Nobel's will, but in light of the continuing expansion of scientific enterprise, possibly far exceeding any 1968-era predictions, it may be time to reconsider its 1968 decision to disallow new prizes, while taking care not to dilute existing ones.

The value of the Nobel Prize in affirming the role of public support for research should not be underestimated. The arguments that scientists have with the prize do not detract from this. Although other international science prizes exist, none are comparable in public stature. Ultimately the greatest value of such prizes rests in the case they make for scientific research to the wider public and not in the money or prestige heaped on a fortunate few, deserving though they may be. It would be a shame if the dissatisfaction of some scientists threatened the good will the Nobel Prizes bestow on science as a whole. 